\title{
Characterization of Switchable Rectangular Ring FSS with Non Coupled Parallel Metallic Strips for Multi Band and Dual Polarized Applications Using WCIP Method
}

\author{
R. Saidi, ${ }^{1}$ M. Titaouine,,${ }^{1,2}$ A. Djouimaa, ${ }^{1}$ \\ ${ }^{1}$ Department of Electronics, Faculty of Technology, University of Batna 2, Batna 05000, Algeria \\ ${ }^{2}$ Laboratory of Materials and Electronic Systems LMSE, Faculty of Sciences and Technology \\ University of Bordj Bou Arreridj, University, El Anasseur 34625, Algeria \\ saidiraouia@gmail.com, mohammedtitaouine@gmail.com, \\ awatefdjouimaa@gmail.com \\ T. R. Sousa, ${ }^{3}$ A. Gomes Neto, ${ }^{3}$ \\ ${ }^{3}$ Group of Telecommunications and Applied Electromagnetism, \\ GTEMA, Federal Institute of Education Science and Technology of Paraiba, IFPB, Av. 1 de Maio, \\ 720, Joao Pessoa, PB, CEP 58015-430, Brazil \\ thayuan.rolim@gmail.com, alfredogomesjpa@gmail.com \\ K. Bencherif, ${ }^{1}$ \\ ${ }^{1}$ Department of Electronics, Faculty of Technology, University of Batna 2, Batna 05000, Algeria \\ bencherifka@gmail.com \\ H. Baudrand, ${ }^{4}$ \\ ${ }^{4}$ University of Toulouse; INPT, UPS; LAPLACE; ENSEEIHT, 2 Rue Charles Camichel, Toulouse \\ Cedex 7, BP 7122, F-31071, France \\ henri.baudrand@yahoo.fr
}

\begin{abstract}
In this paper, a rectangular metallic ring FSS (Frequency Selective Surfaces) with parallel metallic strips for multi band applications are characterized by the WCIP (Wave Concept Iterative Procedure) method. The proposed FSS structures are made of metallic elements that are simultaneously independent in the spatial domain and uncoupled in the spectral domain. Thus the FSS multiband behavior can be achieved only by a building process based on independent metallic elements in both domains. The resonant characteristics of the proposed FSS are determined. The structure shows three resonant frequencies when the incident wave is $x$-polarized and three resonant frequencies when the source is polarized in the orthogonal direction. A variation in the length of the strips and the rectangular ring allows a fine tuning in the FSS resonant frequencies to give raise to tuned bandwidths. The suppression of a resonant frequency can be insured either by the elimination of the responsible metallic strip or by the insertion of a PIN diode switch. Three FSS structures are manufactured and characterized. The FSS bandwidth can be enhanced in both perpendicular FSS exciting source directions by only varying the strips lengths and the ring dimensions to the corresponding resonant frequencies. A good agreement is obtained when the WCIP results are compared with the simulations of COMSOL Multiphysics software and experiment.
\end{abstract}

Index Terms - WCIP method, FSS structures, transmission coefficient, metallic strips, rectangular ring, resonant frequency, PIN diode, enhanced bandwidth. 


\section{INTRODUCTION}

Along the decades, the increased demand for telecommunication systems services, due to the technological progress encouraged the development of new frequency selective surfaces having multiband applications and smaller sizes [1], [2]. The applications of frequency selective surfaces (FSS) are varied; in the microwave the FSS are exploited to make a reflector antenna more efficient, another application in this wavelength range is the radome. In the far-infrared region, periodic screens are used as polarizers, beam splitters, as well as mirrors for improving the pumping efficiency in molecular lasers [3], [4].

Many previous works have presented FSSs for multi-band and dual-polarization applications. In concentric rectangular rings FSS the insertion of one more ring results in the addition of one resonant frequency in both perpendicular directions. To decrease the metallic surface of the FSS maintaining the multi band and dual polarization characteristics the quasi-rectangular open-rings is introduced [5], [6], [7], [8], [9]. In the U-shaped FSS the two resonant are controlled by the strips lengths [5]. To increase the number of resonances without altering the FSS interface another FSS layer is added to end with a double layered FSS structure [10], [11]. Theses FSS structures suffers from being not under the total control of the users since changing one or many FSS metallic elements dimensions at a time leads to a non prior known frequency response unless a deep parametric study is done. Moreover, with these FSS it is not possible to build the desired frequency response in all the cases. Thus an equivalent circuit capable of modeling these FSS for all the cases is hard to determine.

To overcome the previous FSS drawbacks, in this paper the presented FSS structure is made of parallel non coupled metallic strips with one rectangular metallic ring acting as a multi-band and a dual polarized filter with three resonant frequencies for an x-polarized source and three resonant frequencies for a y-polarized source. The proposed structure is composed of uncoupled metallic strips in such a way that a resonant frequency is added (or suppressed) from the FSS frequency response by only adding (or suppressing) its resonant frequency responsible strip or ring. Hence this independence of the metallic elements in the spatial domain leads to the metallic elements behaviors independence in the frequency domain. Many simulations have been simulated by the Comsol Multiphysics software, HFFS software, and the WCIP method to end with the FSS final arrangement with uncoupled metallic elements allowing building the FSS frequency response from individual metallic elements frequency responses in two polarizations with the same facilities of the spatial domain.

In this paper, a rectangular metallic ring FSS with non coupled parallel metallic strips for multi band applications is proposed. The presented FSS shows three resonant frequencies at about $6 \mathrm{GHz}, 8.25$ $\mathrm{GHz}$ and $10.5 \mathrm{GHz}$ when the incident wave is $x$-polarized and three resonant frequencies at about 6 $\mathrm{GHz}, 8.75 \mathrm{GHz}$ and $10.5 \mathrm{GHz}$ when the source is $y$-polarized. The scattering parameters of the 
proposed structures are characterized by the WCIP (Wave Concept Iterative Procedure) method, COMSOL Multiphysics and measurements. In the proposed FSS structure each resonant frequency can be deleted by only eliminating the corresponding metallic strip. For more flexible FSS selectivity and electronically controlled FSS a PIN diode switch is inserted at the center of the strip to bring the corresponding resonant frequency higher so that it is seen as eliminated. Moreover, the presented FSS bandwidth can be enhanced by independently and simultaneously varying the strips length and the ring dimensions to the appropriate values.

The WCIP method (Wave Concept Iterative Procedure) gets its originality from its ease of implementation since it is based on two essential equations. The former is defined in the spatial domain and it is a description of the interface using the boundary conditions. Whereas the letter is described in the modal domain and it summarizes the reflection of the diffracted waves getting out of the interface from the modes closing impedances due to the closing conditions. The WCIP speed is obtained from the systematic use of the fast modal transform pair FMT/FMT ${ }^{-1}$ [6] integrating in its procedure the two dimensional fast Fourier transform pair FFT/FFT ${ }^{-1}$.

The WCIP method is used for obtaining simulation results of the single interface multilayer FSS [12], [13]. Three proposed FSS structures are manufactured and characterized. WICIP results are compared to results simulated by COMSOL Multiphysics $4.3 \mathrm{v}$ software package and experimental measurements and a good agreement is recorded.

\section{WCIP FORMULATION}

The WCIP method is based on the transverse-formulation and information obtained at the interfaces [14]. Multiple reflection process is started using the initial conditions and stopped once convergence is reached. The diffraction operator $\widehat{S} \Omega$ and the reflection operator $\widehat{\Gamma}$ relate the incident and diffracted waves in the spatial and spectral domain respectively and govern the iterative procedure [14]. In the WCIP method, the FSS analysis is reduced to that of the unit cell. Fig. 1.a shows an FSS with arbitrary shape excited with a normal incident plane wave along the negative $z$ direction. The dashed lines are the hypothetical periodic walls. An elementary cell is shown in Fig. 1(b).

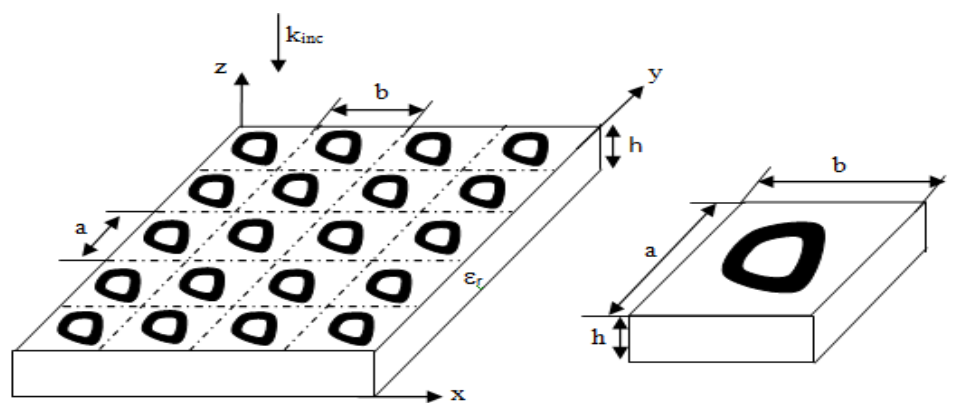

(a)

(b)

Fig.1. Arbitrary FSS: (a) Geometry of the FSS and (b) FSS unit cell. 
The incident and the reflected waves can be obtained from the transverse electric and magnetic fields as [15]-[18]:

$$
\begin{aligned}
& \overrightarrow{\mathrm{A}}_{\mathrm{i}}=\frac{1}{2 \sqrt{\mathrm{Z}_{0 \mathrm{i}}}}\left(\overrightarrow{\mathrm{E}}_{\mathrm{Ti}}+\mathrm{Z}_{0 \mathrm{i}} \overrightarrow{\mathrm{J}}_{\mathrm{i}}\right) \\
& \overrightarrow{\mathrm{B}}_{\mathrm{i}}=\frac{1}{2 \sqrt{\mathrm{Z}_{0 \mathrm{i}}}}\left(\overrightarrow{\mathrm{E}}_{\mathrm{Ti}}-\mathrm{Z}_{0 \mathrm{i}} \overrightarrow{\mathrm{J}}_{\mathrm{i}}\right)
\end{aligned}
$$

$\mathrm{Z}_{0 \mathrm{i}}$ indicates the characteristic impedance of medium 1 or medium 2 [14].

The diffracted waves $\overrightarrow{\mathrm{B}}_{i}^{(\mathrm{k})}$ at the $\mathrm{k}^{\text {th }}$ iteration are obtained from the incident waves $\overrightarrow{\mathrm{A}}_{i}^{(\mathrm{k})}$ by [6]:

$$
\overrightarrow{\mathrm{B}}_{\mathrm{i}}^{(\mathrm{k})}=\widehat{\mathrm{S}}_{\Omega} \overrightarrow{\mathrm{A}}_{\mathrm{i}}^{(\mathrm{k})}
$$

$\widehat{\mathrm{S}}_{\Omega}$ is the diffraction operator at the interface $\Omega$ containing the FSS circuit. It depends on the boundary conditions of each domain and the geometry of the structure. The diffraction phenomena is seen in the modal domain and it takes place once one incident wave strikes the interface and as a result an infinity of TE (Transverse Electric) and TM (Transverse Magnetic) modes appears due to the presence of a metal-dielectric discontinuity. Hence equation (3) is considered as a dynamic equation since if the FSS structure pattern is changed this equation has to be also changed due to the presence of the diffraction operator $\widehat{S}_{\Omega}$ depending on the physical interface containing the electric circuit.

Once the diffracted waves appear, they are reflected by the modes closing impedances at the closing wall representing the two ends of the hypothetical rectangular periodic waveguide containing the FSS unit cell. The incident waves $\overrightarrow{\mathrm{A}}_{\mathrm{i}}^{(\mathrm{k}+1)}$ of the $(\mathrm{k}+1)^{\text {th }}$ iteration can be calculated by [6]:

$$
\overrightarrow{\mathrm{A}}_{\mathrm{i}}^{(\mathrm{k}+1)}=\widehat{\Gamma}_{\mathrm{i}} \mathrm{B}_{\mathrm{i}}^{(\mathrm{k})}+\overrightarrow{\mathrm{A}}_{0}
$$

Where $\hat{\Gamma}_{\mathrm{i}} \mathrm{B}_{\mathrm{i}}^{(\mathrm{k})}$ represents the reflected waves with $\widehat{\Gamma}_{\mathrm{i}}$ being the reflection operator defined in the modal domain.

The FMT/FMT ${ }^{-1}$ transform pair given in [6] allows the transition between the spatial domain and the modal domain.

Equation (4) is structure independent and it is seen as a static equation. Hence the only parameter that depends on the structure is the diffraction operator. Thus when changing the structure all the algorithm parameters remain the same except the diffraction operator giving to the WCIP method another potential favor in studying planar circuits as compared to other methods such as the method of moments where if the structure is changed all the parameters constituting its algorithm have to recalculated. 
A multiple reflection procedure is started using the initial conditions and stopped once the convergence is reached. Thus, the tangential electric and magnetic fields can be calculated as [12], [13], [19]-[21]:

$$
\begin{gathered}
\vec{E}_{i}=\sqrt{Z_{0 i}}\left(\overrightarrow{A_{1}}+\vec{B}_{i}\right) \\
\vec{J}_{i}=\left(1 / \sqrt{\left.Z_{0 i}\right)}\left(\vec{A}_{i}-\vec{B}_{i}\right)\right.
\end{gathered}
$$

The WCIP method adopted in this paper doesn't account for the losses in the FSS structures. The only source of losses is the limited number of used modes due to the meshed interface. Theoretically the convergence cannot be obtained at the resonance since the waves at this state are reflected back and forth by the interface and the closing walls as if they are imprisoned. But the losses caused by the limitation in the number of used TE and TM modes in the analysis forces the convergence to exist at structure resonance. The use of the medium impedance $\mathrm{Z}_{0 \mathrm{i}}$ can be replaced by any impedance $\mathrm{Z}_{0}[6]$ with $\mathrm{Z}_{01}=\mathrm{Z}_{02}=\mathrm{Z}_{0}$. It is initially used as medium impedance to provide some physical sight on its value.

\section{THE PARALLEL METALLIC STRIPS WITH RECTANGULAR METALLIC RING FSS}

\section{DESIGN}

The proposed structure is a parallel non coupled metallic strips with one rectangular metallic ring, shown in Fig. 2(a). The manufactured FSS of $20 \mathrm{~cm} \times 20 \mathrm{~cm}$ size is printed on a fiber glass (FR-4) substrate with dielectric constant of $\varepsilon_{\mathrm{r}}=4.4$ and a thickness of $h=1.5 \mathrm{~mm}$. The remaining FSS dimensions shown in Fig. 2(b) representing the FSS unit cell are: $L_{1}=10.45 \mathrm{~mm}, L_{2}=7.27 \mathrm{~mm}, L_{3}=$ $11.36 \mathrm{~mm}, L_{4}=9.45 \mathrm{~mm}, L_{5}=7.27 \mathrm{~mm}, W_{1}=W_{2}=W_{4}=W_{5}=1 \mathrm{~mm}, W=1 \mathrm{~mm}$ and $W_{3}=11.81 \mathrm{~mm}$.

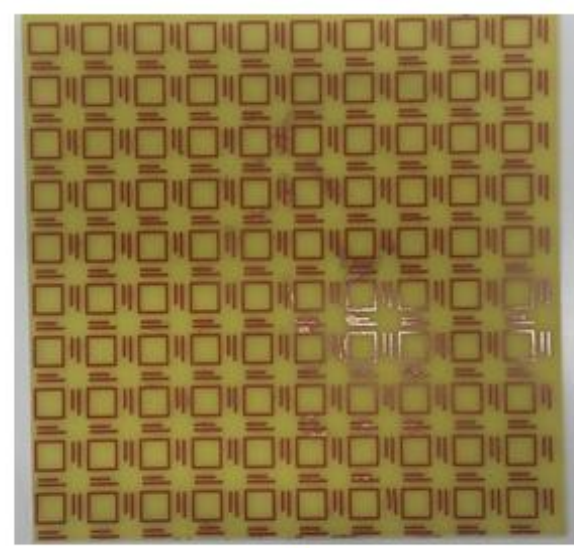

(a)

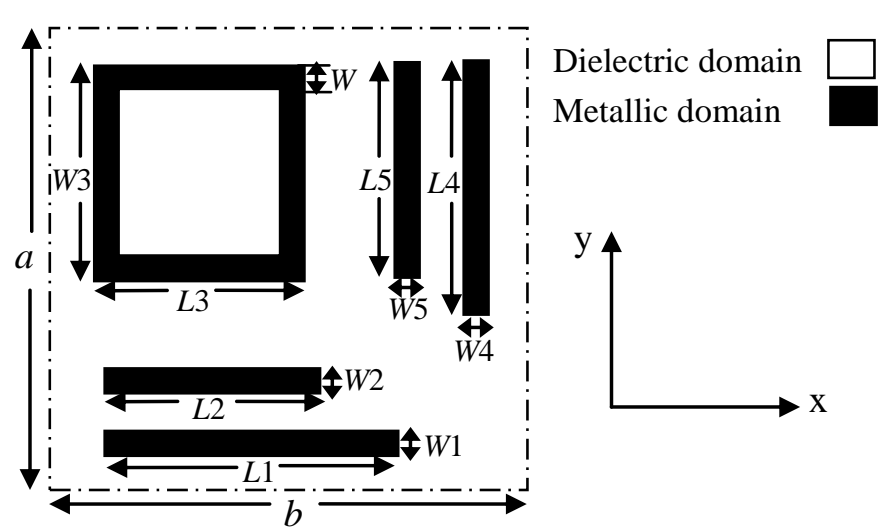

(b)

Fig. 2. The rectangular metallic ring FSS with parallel metallic strips: (a) Realized FSS with an array of $10 \times 10$ unit cells and (b) FSS unit cell geometry.

Brazilian Microwave and Optoelectronics Society-SBMO Brazilian Society of Electromagnetism-SBMag received 08 Sep 2017; for review 08 Sep 2017; accepted 15 Mar 2018 (C) 2018 SBMO/SBMag (cc) BY
ISSN 2179- 


\section{Results and analysis}

To validate the implemented WCIP method results for no need to more improvement to accelerate convergence for the used grid and stopping iterative procedure in addition to mastering Comsol Multiphysics for planar FSS problems, the scattering parameters of the structure shown in figure 2 are obtained by the WCIP method. A grid of $100 \times 100$ pixels is used to describe the FSS interface and the iterative process is stopped after 350 iterations. As shown in Fig .3(a) the resonant frequencies are about $6 \mathrm{GHz}, 8.25 \mathrm{GHz}$ and $10.5 \mathrm{GHz}$ when the incident wave is $\mathrm{x}$-polarized and $6 \mathrm{GHz}, 8.75 \mathrm{GHz}$ and $10.5 \mathrm{GHz}$ when the source is $y$-polarized as shown in the Fig. 3(b).

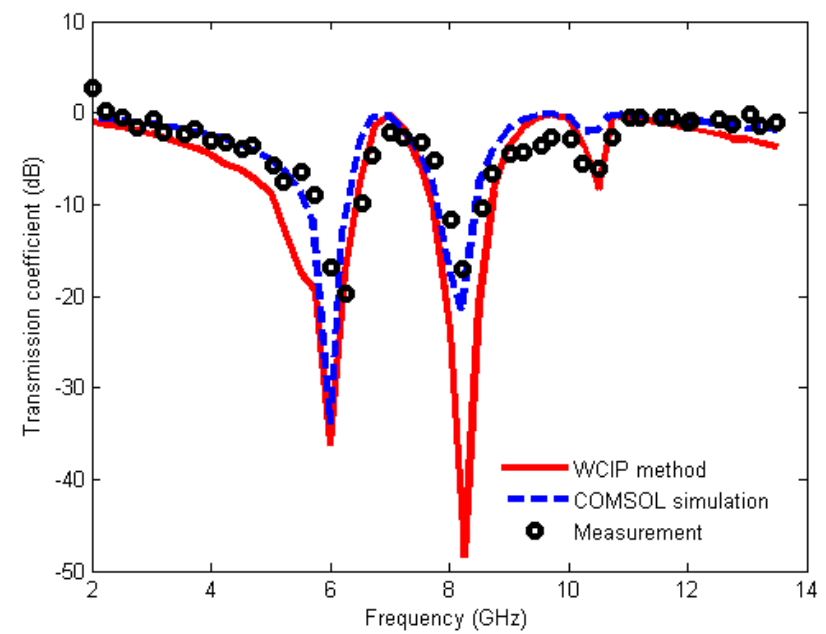

(a)

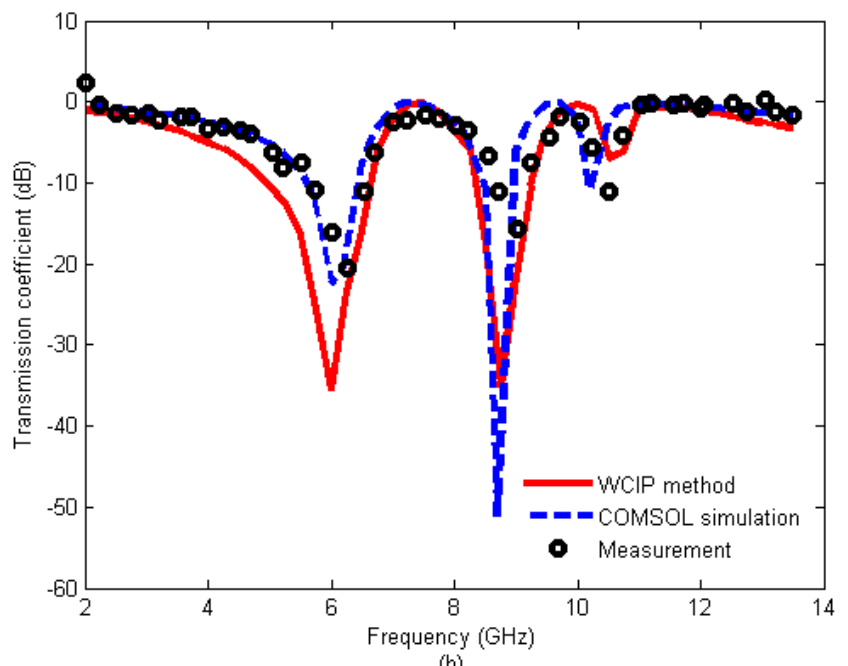

(b)

Fig. 3. Simulated and measured transmission coefficient versus the operating frequency for:

(a) The $x$-polarized source (b) The $y$-polarized source

In the next part and using the implemented WCIP method, the characteristic of the presented FSS as being composed of uncoupled FSS elements is independently verified for each metallic strip. It is shown that the variation of one strip length maintaining the remaining FSS elements dimensions unchanged alter only the length varied strip resonance without affecting the other strips resonances. 


\section{A. Variation of the strip length $L_{1}$}

The results concerning the transmission coefficients for different values of the length of the strip $L_{1}$ of Fig. 1(b) obtained by the WCIP method and the Comsol software are given in Fig. 4.

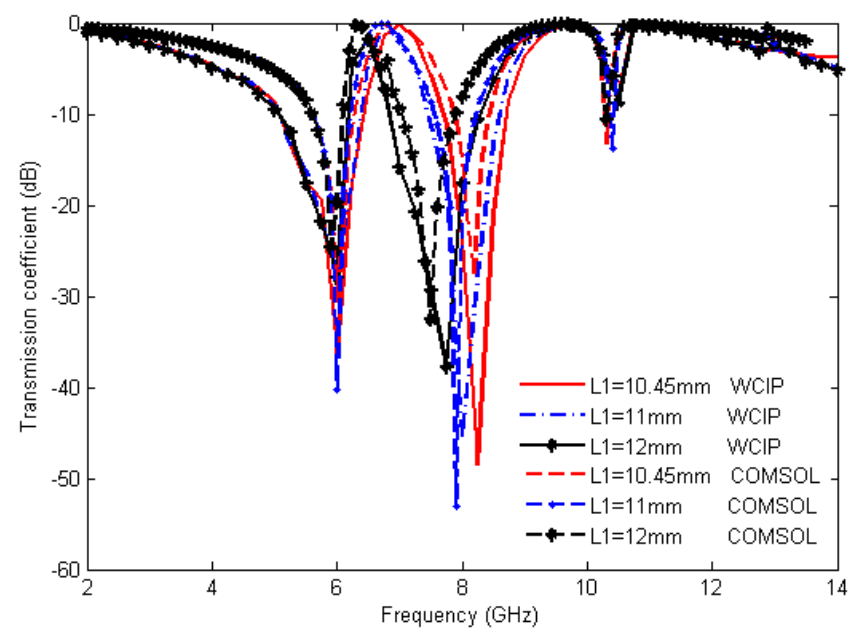

Fig. 4. Transmission coefficient versus frequency for different values of $L_{1}$ for an $x$-polarized source WCIP results and COMSOL results.

Observing figure 4 , it is noted that when using the WCIP method the strip length $L_{1}$ increases its corresponding resonant frequency decreases from $8.25 \mathrm{GHz}$ to $7.75 \mathrm{GHz}$ for values of $L_{1}$ equal to $10.45 \mathrm{~mm}$ and $12 \mathrm{~mm}$ respectively in the x polarization. The corresponding resonant frequencies using Comsol multiphysics software decrement from $8.2 \mathrm{GHz} 7.5 \mathrm{GHz}$. The relative error between the WCIP method results and Comsol Multiphysics software in determining the resonant frequencies are $0.61 \%, 1.26 \%$ and $3.33 \%$ when $L_{1}$ equals to $10.45 \mathrm{~mm}, 11 \mathrm{~mm}$ and $12 \mathrm{~mm}$ respectively. The principle characteristic of the presented FSS structure is shown by the fact that the two resonant frequencies recorded at $6 \mathrm{GHz}$ and $10.5 \mathrm{GHz}$ are not altered since the strips and the ring are not coupled.

\section{B. Variation of the strip length $L 2$}

The transmission coefficients of the structure as a function of the operating frequency, corresponding to different values of $L_{2}$ are shown in Fig. 5. 


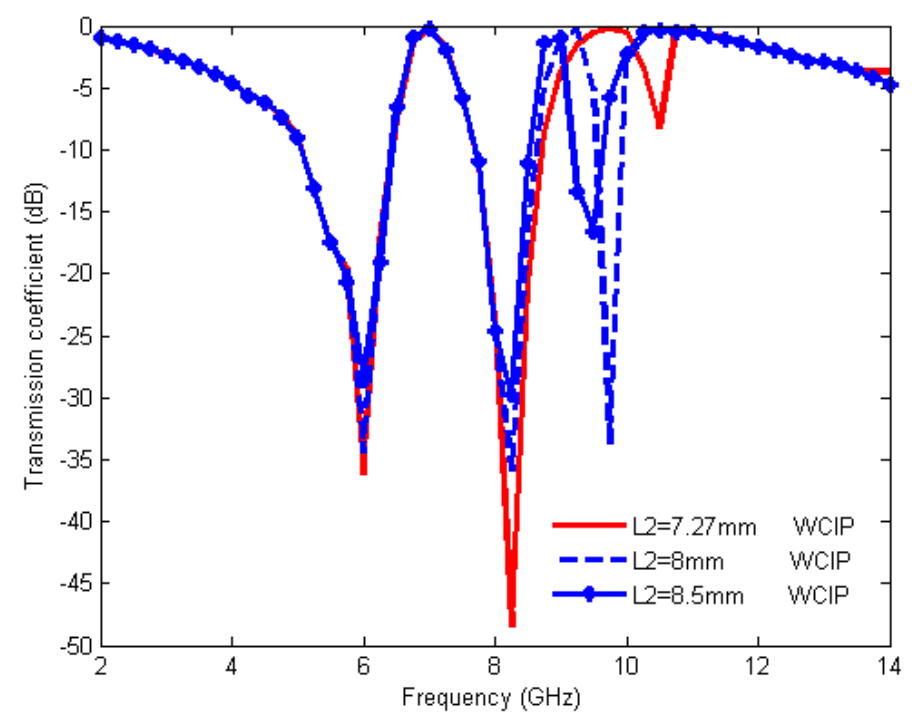

Fig. 5. The transmission coefficient versus the frequency for $L_{2}=7.27 \mathrm{~mm}$ until $8.5 \mathrm{~mm}$ for an $x$-polarized source.

When the length $L_{2}$ increases the resonance frequency decreases, giving a value of $10.5 \mathrm{GHz}$ for $L_{2}$ equal to $7.27 \mathrm{~mm}$ and $9.5 \mathrm{GHz}$ for a value of $L_{2}$ equal to $78.5 \mathrm{~mm}$ in the $x$ polarization.

In the y polarization the effect of varying the lengths $L_{1}$ and $L_{2}$ on the resonant frequencies is not observed.

\section{Variation of the strip length $L_{4}$}

As shown in Fig. 6, the resonant frequency of the structure is decreasing when $L_{4}$ is increasing, taking values of $8.75 \mathrm{GHz}$ for $L_{4}=9.45 \mathrm{~mm}, 8.5 \mathrm{GHz}$ for $L_{4}=10.25 \mathrm{~mm}$ and $8 \mathrm{GHz}$ for $L_{4}=11 \mathrm{~mm}$.

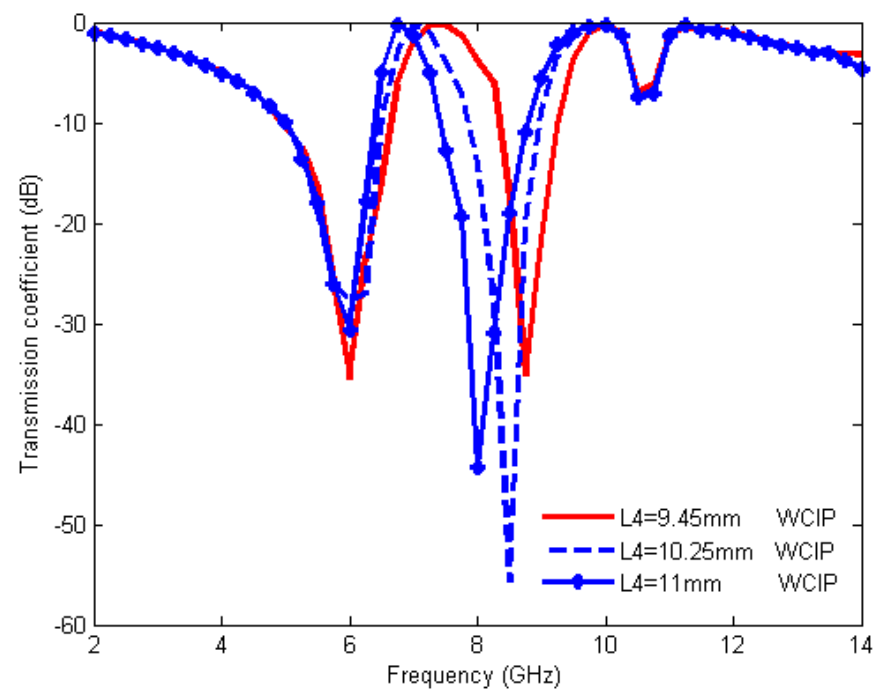

Fig. 6. The transmission coefficient versus the frequency for different values of $L_{4}$ for a $y$-polarized source. Brazilian Microwave and Optoelectronics Society-SBMO received 08 Sep 2017; for review 08 Sep 2017; accepted 15 Mar 2018 Brazilian Society of Electromagnetism-SBMag (c) 2018 SBMO/SBMag 
D. Variation of the strip length $L 5$

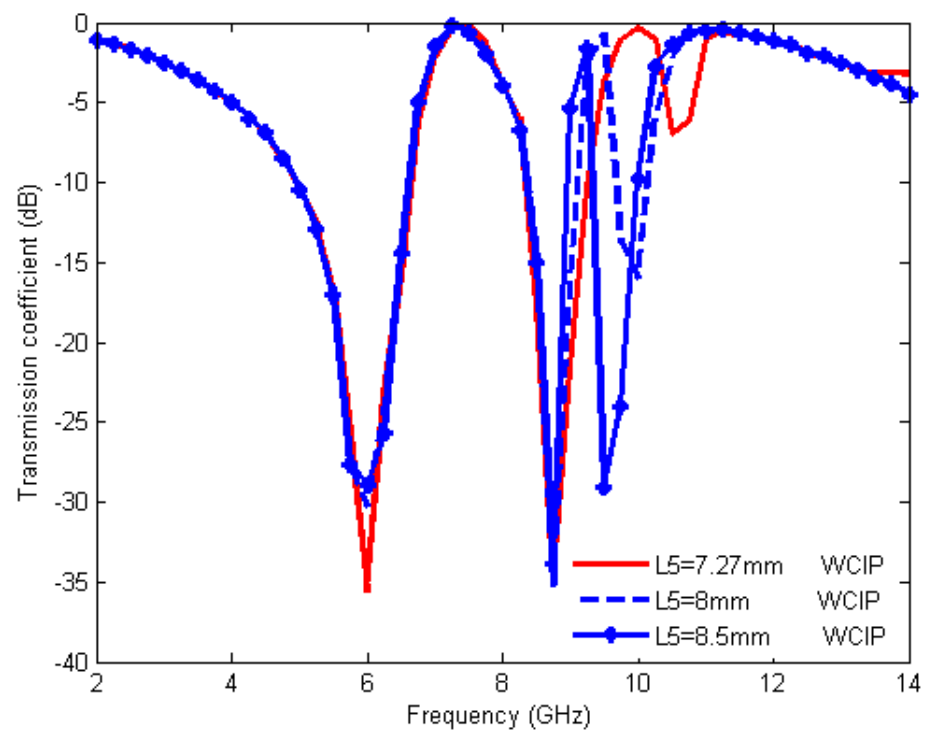

Fig. 7. The transmission coefficient versus the frequency for $L_{5}=7.27 \mathrm{~mm}$ until $8.5 \mathrm{~mm}$ for a $y$-polarized source.

The transmission coefficients as a function of the operating frequency, corresponding to different values of $L_{5}$ are shown in Fig. 7. When the length $L_{5}$ increases from $7.27 \mathrm{~mm}$, to $8 \mathrm{mmm}$ until $8.5 \mathrm{~mm}$ the resonance frequency decreases, from $10.5 \mathrm{GHz}$ until $9.75 \mathrm{GHz}$.

In the case of an $x$-polarized source of excitation, the effect of varying $L_{4}$ and $L_{5}$ is not recorded. Hence, the presented FSS structure is based on non coupled parallel metallic strips. As a result the proposed FSS resonant frquencies can be independently adjusted by only varying the corresponding strips lengths.

\section{E. Switchable FSS}

Another way of confirming that a strip is uncoupled with the remaining structure elements is by physically eliminating it without affecting the remaining FSS elements frequency response. To confirm the non coupling of the strip $L_{5}$ and its presence lead to inserting the highest resonant frequency $10.5 \mathrm{GHz}$ of the structure in the y polarization, it is definitively eliminated, taking the remaining dimensions equal to: $L_{1}=10.58 \mathrm{~mm}, L_{2}=7.50 \mathrm{~mm}, L_{3}=11.32 \mathrm{~mm}, L_{4}=9.70 \mathrm{~mm}, W_{3}=11.82 \mathrm{~mm}$ $W=1 \mathrm{~mm}$. The structure was manufactured and measured as shown in Fig. 8. 

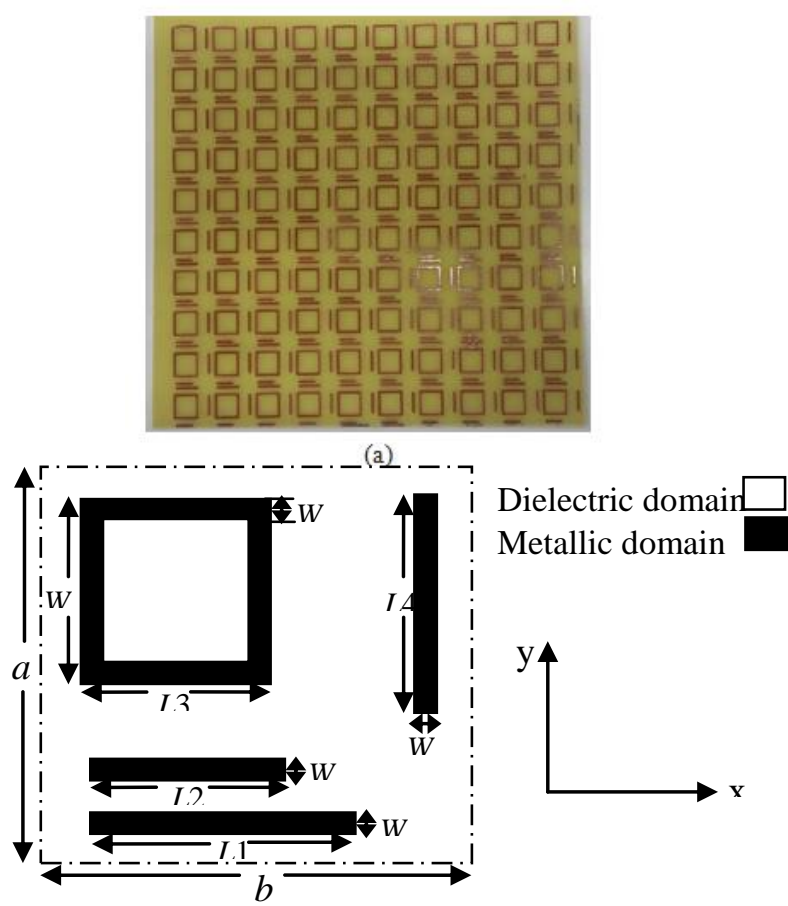

(b)

Fig. 8. The rectangular metallic ring FSS with parallel metallic strips:

(a) Realized FSS with an array of $10 \times 10$ unit cells and (b) FSS unit cell geometry without $L_{5}$.

Fig. 9 presents the transmission power of the structure of Fig. 8. It is clear that the highest resonant frequency at $10.5 \mathrm{GHz}$ is eliminated when the structure is y polarized while the other resonant frequencies are maintained fixe in both polarization $x$ and $y$.

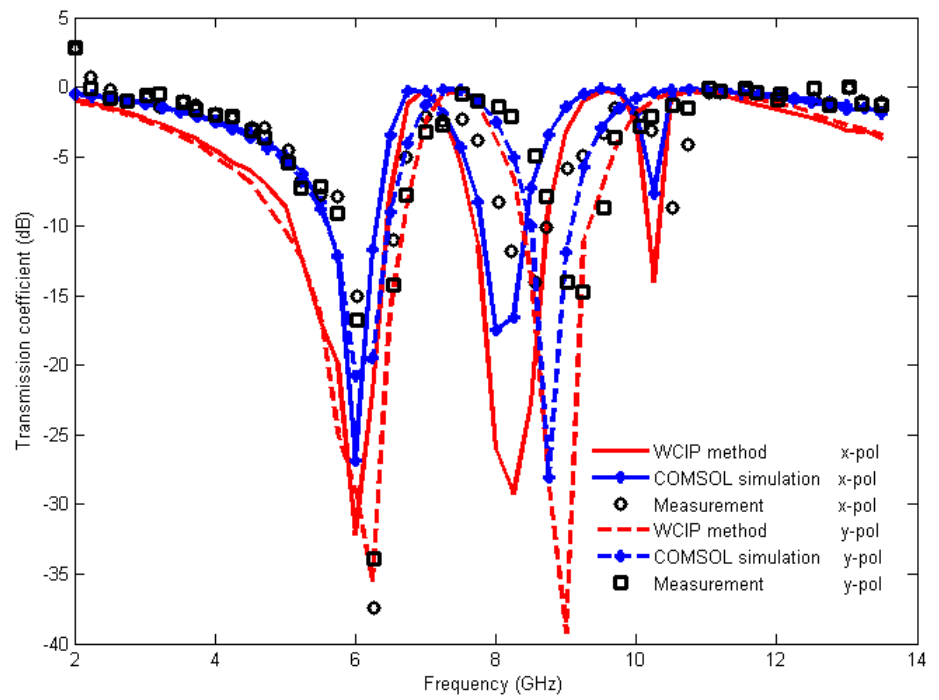

Fig. 9. Simulated and measured results for the transmitted power of the parallel metallic strips with rectangular metallic ring FSS versus frequency according to the $x$ and $y$ polarizations. 
To take benefit of both cases of the presence of the highest resonant frequency and its elimination a switchable FSS is necessary. The strip elimination is achieved by pushing its corresponding resonance out of the operating frequency window ranging from $2 \mathrm{GHz}$ to $14 \mathrm{GHz}$. Even though it is not physically suppressed its behavior is eliminated. Thus switchable FSS consists in the addition or the elimination of a metallic strip via the insertion of an ideal ON-OFF states switch in the center of a strip to bring higher its corresponding resonant frequency, $2 f_{r}$. The ideal switch is replaced by a gap of dimensions $1 \mathrm{~mm} \times 1 \mathrm{~mm}$ as shown in Fig. 10 where the switch is inserted in the center of the strip $L_{2}$. The structure is fabricated and characterized.

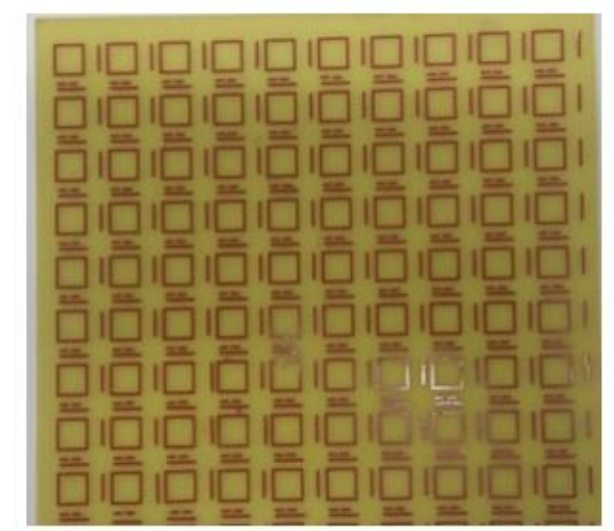

(a)

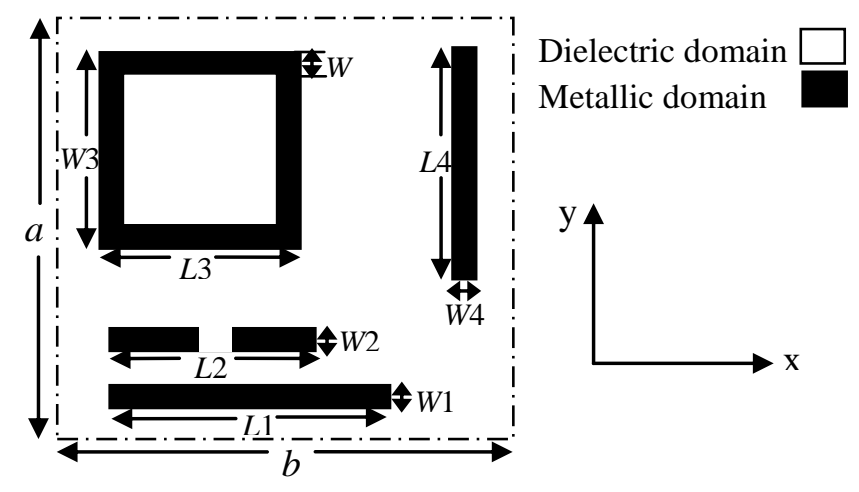

(b)

Fig.10. The rectangular metallic ring FSS with parallel metallic strips:

(a) Realized FSS with an array of $10 \times 10$ unit cells and (b) FSS unit cell geometry with a gap on the strip length $\mathrm{L}_{2}$.

The Transmission power of the structure of Fig. 10 is shown in Fig. 11. It shows only two resonances at about $6.25 \mathrm{GHz}$, and $8.25 \mathrm{GHz}$ according to the $x$ polarization with a clear elimination of the third resonant frequency and two resonances at about $6 \mathrm{GHz}$, and $9 \mathrm{GHz}$ according to the $y$ polarization. 


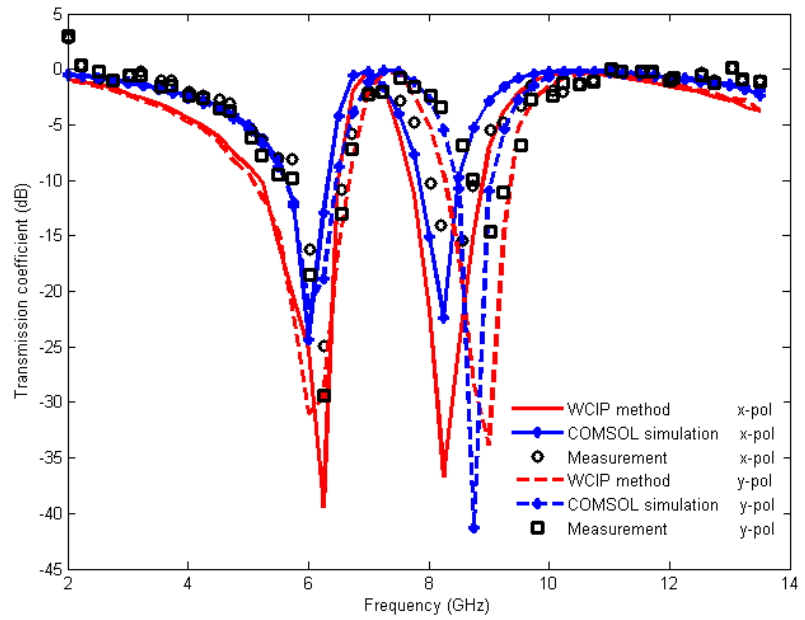

Fig. 11. Simulated and measured transmission coefficient versus the frequency for the $x$ and $y$ polarizations.

When PIN diodes are used to operate as RF/microwaves switches, they are modeled as a simple resistance in the forward biasing case and as a resistance in parallel with a capacitor for the reverse biasing diode case. To account for PIN diodes insertion in the WCIP method an impedance diffraction operator is first determined by the use of the diodes equivalent impedance as surface impedance in the domain occupied by the diodes as in [20]. Thus the diode FSS structures consist of four domains with the addition of the diodes domain. In fig.12 the metallic strip FSS with a diode reversely biased at $30 \mathrm{~V}$ and with a diode replaced by a gap the transmission coefficient of is shown representing WCIP results and HFSS results. The diode is reversely polarized at $-30 \mathrm{~V}$ and it is modeled as a resistance of $4 \mathrm{k} \Omega$ in parallel with a capacitor of $0.052 \mathrm{pF}$ and it can be modeled as a resistance of $1 \Omega$ when it is forward biased [22]. The transmission coefficient of the metallic strip FSS with a diode inserted at the strip center is shown in fig. 13. 


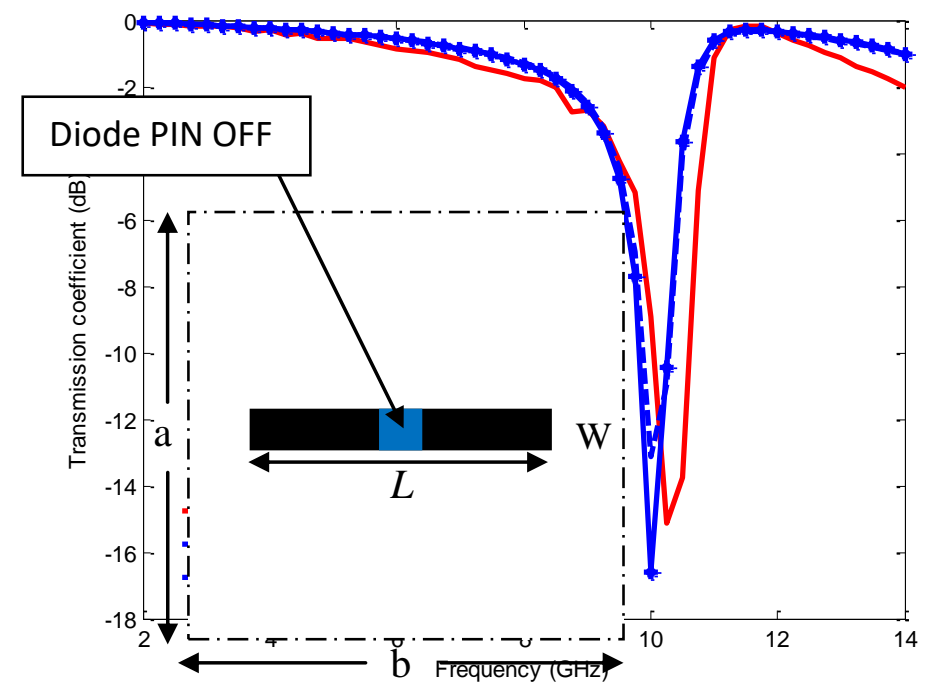

Fig. 12. Simulated transmission coefficient versus the frequency for the $x$-polarized source exciting a metallic strip FSS with a diode reversely biased at the strip center.

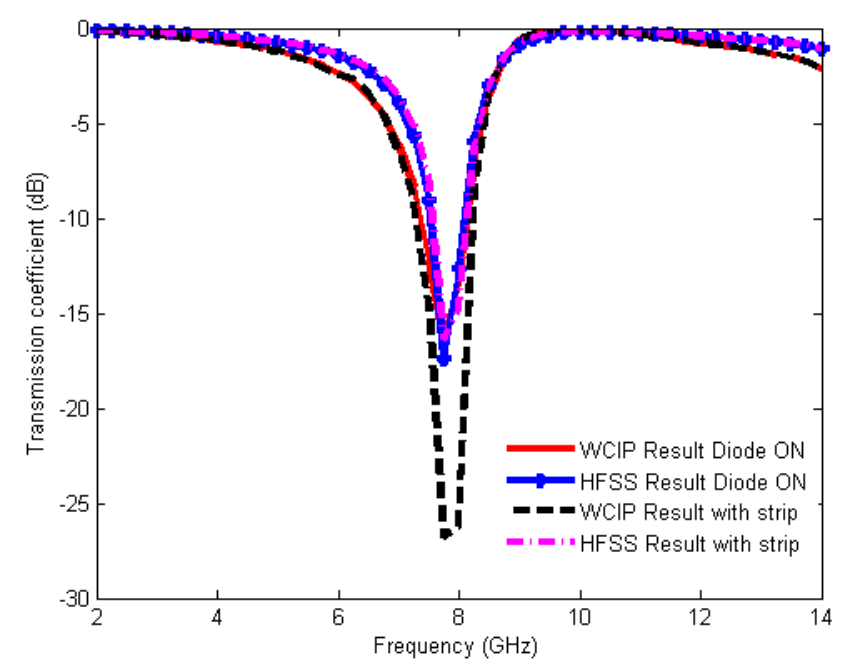

Fig. 13. Simulated transmission coefficient versus the frequency for the $x$-polarized source exciting a metallic strip FSS with a diode forward biased at the strip center.

Figs. 12 and 13 show a good agreement between an ideal ON/OFF switch and a PIN diode switch. Thus the presented measurement of fig. 11 when a gap is inserted can be used to confirm the OFF state PIN diodes presence since the metallic strips in the parallel metallic strip FSS presented are not coupled. 


\section{BANDWIDTH ENHANCEMENT AND TUNABILITY}

The principal advantage of the presented FSS based on non coupled parallel metallic strips in easily enhancing the FSS bandwidth can arise when adjusting independently each of the strips length to give rise to the desired resonant frequency so that the resulting transmission power posses the desired enhanced bandwidth. In addition the rectangular metallic ring dimensions can also used to bring its corresponding resonant frequency to improve the FSS bandwidth. The FSS of fig. $2 b$ is taken as a reference structure to design an enhanced band FSS. At $-3 \mathrm{~dB}$ of the transmission power it has rejecting bandwidths of $3.35 \mathrm{GHz}, 1.76 \mathrm{GHz}$ and $0.46 \mathrm{GHz}$ centered at resonant frequencies respectively when the source is $x$ polarized. In the case of the $y$ polarized source the rejecting bandwidths are $3.70 \mathrm{GHz}, 1.65 \mathrm{GHz}$ and $0.57 \mathrm{GHz}$. At $-10 \mathrm{~dB}$ of the transmission power two rejecting bands exist in both source polarizations with bandwidths of $1.37 \mathrm{GHz}, 1.021 \mathrm{GHz}$ for the $x$ polarized source and $1.71 \mathrm{GHz}$ and $0.92 \mathrm{GHz}$ when the source is $y$ polarized. Fig. 14 shows the variation of the transmission coefficient as a function of the operating frequency for different values of the metallic strip of length $L_{2}$ resulting in decreasing the FSS higher resonant frequency. For $L_{2}$ equals to $10.45 \mathrm{~mm}$, its corresponding resonant frequency intervenes to enhance the bandwidth situated at $8.25 \mathrm{GHz}$ for the $x$ polarized FSS. Thus bandwidth presented initially at this frequency is enlarged to $2.44 \mathrm{GHz}$ at $-3 \mathrm{~dB}$ and $1.42 \mathrm{GHz}$ at $-10 \mathrm{~dB}$ to result to a band enhancement of $38.64 \%$ at $-3 \mathrm{~dB}$ and $39.08 \%$ at $-10 \mathrm{~dB}$. Another mean of enhancing the improved band at $8.25 \mathrm{GHz}$ is a decrease in the metallic ring dimension $L_{3}$ to result in moving higher its corresponding resonant frequency as shown in fig. 15. When $L_{3}$ takes the value of $5 \mathrm{~mm}$ its corresponding bandwidth overlaps with the initial rejecting bandwidth situated at $8.25 \mathrm{GHz}$ to result in enhancing again the rejecting bandwidth situated at $8.25 \mathrm{GHz}$ to $4.10 \mathrm{GHz}$ at $-3 \mathrm{~dB}$ and $1.65 \mathrm{GHz}$ at $-10 \mathrm{~dB}$. Thus a bandwidth improvement of $123.86 \%$ at $-3 \mathrm{~dB}$ and $61.60 \%$ at $-10 \mathrm{~dB}$ as compared to the initial FSS rejecting bandwidth occurring at $8.25 \mathrm{GHz}$ is obtained. 


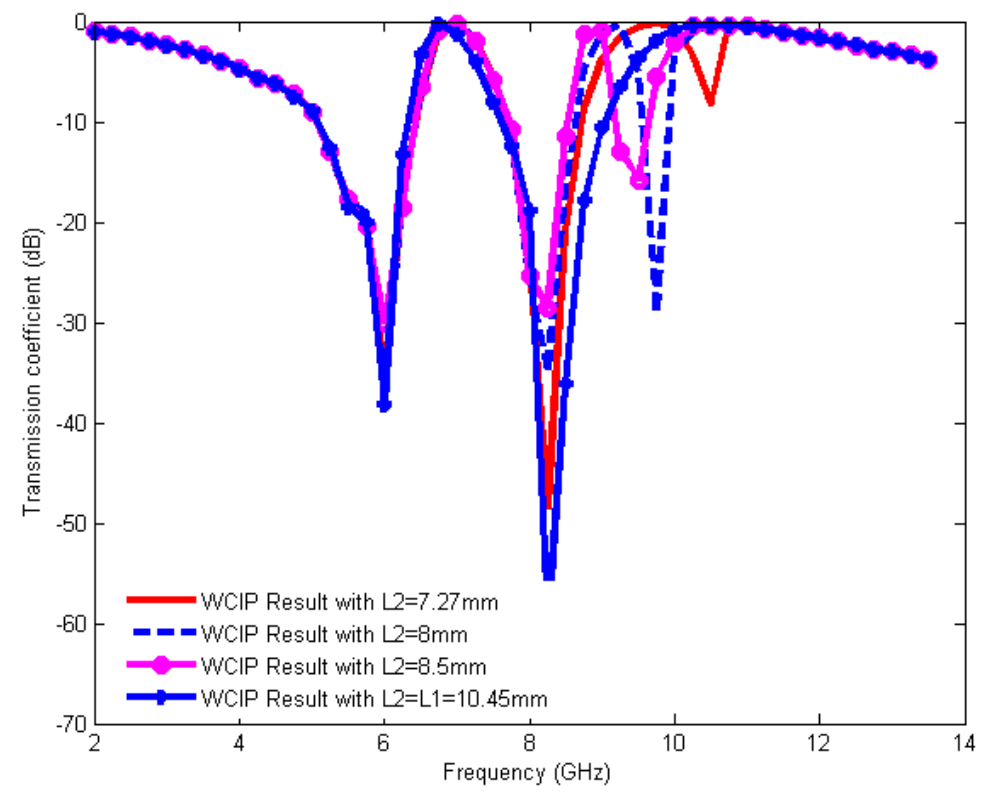

Fig. 14. Transmission coefficient for different increasing values of L2 for $x$-Polarization

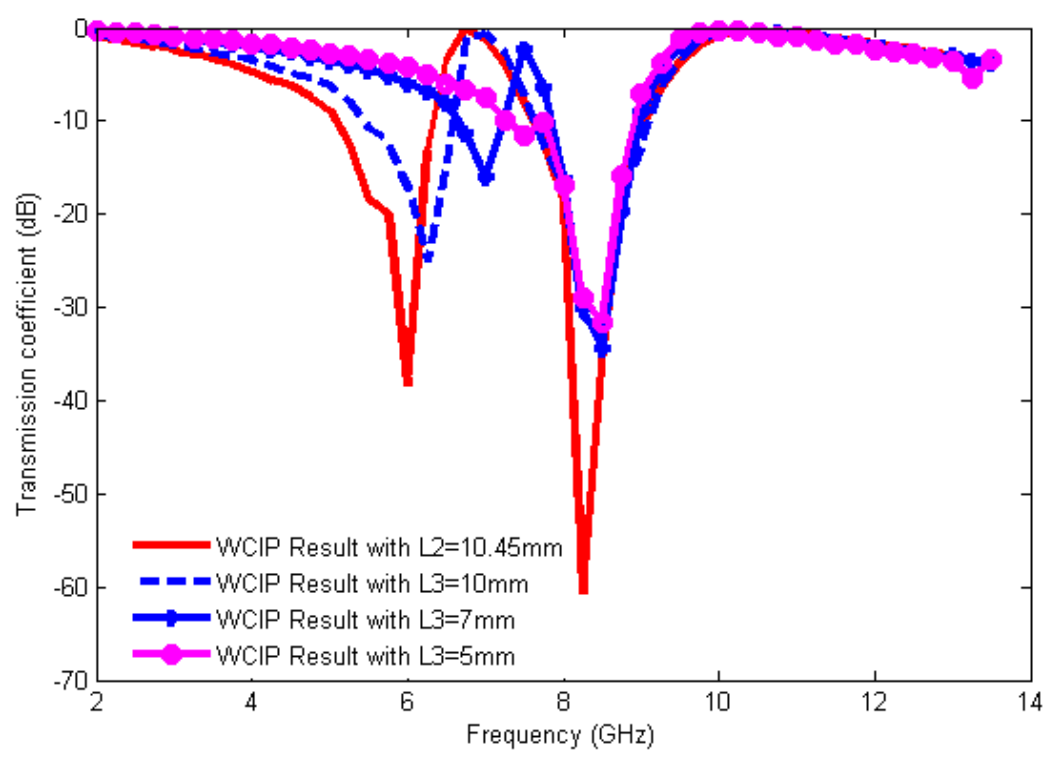

Fig. 15. Transmission coefficient for different decreases values of L3 and fixed L2 $=10.45 \mathrm{~mm}$ for $y$-Polarization

To improve the FSS bandwidth when it is excited with a $y$ polarized source, the strip length $L_{5}$ is increased to $9.45 \mathrm{~mm}$ to take the same value of $L_{4}$ as shown in fig. 16 . A bandwidth of $2.40 \mathrm{GHz}$ at $3 \mathrm{~dB}$ and $1.3 \mathrm{GHz}$ at $-10 \mathrm{~dB}$ is observed. Thus the rejecting bandwidth occurring at a resonant frequency of at $8.25 \mathrm{GHz}$ is enhanced with $45.45 \%$ at $-3 \mathrm{~dB}$ and $41.3 \%$ at $-10 \mathrm{~dB}$ occurs. The obtained $y$ polarized FSS enhanced bandwidth can be improved again by decreasing the ring dimension $W_{3}$ as

Brazilian Microwave and Optoelectronics Society-SBMO Brazilian Society of Electromagnetism-SBMag received 08 Sep 2017; for review 08 Sep 2017; accepted 15 Mar 2018 (C) 2018 SBMO/SBMag 
shown in fig. 17. When $W_{3}$ decreases to $4 \mathrm{~mm}$ the resulting rejecting bandwidth situated at $8.25 \mathrm{GHz}$ is improved to $3.67 \mathrm{GHz}$ at $-3 \mathrm{~dB}$ without being altered for $-10 \mathrm{~dB}$ reference. Hence an overall rejecting bandwidth enhancement of $122.42 \%$ at $-3 \mathrm{~dB}$ occurring at $8.25 \mathrm{GHz}$ is achieved as compared to the initial FSS of fig. 2.

In the proposed FSS the resonant frequency of the enhanced bandwidths can be easily tuned to the desired frequency by varying independently and simultaneously the corresponding strips lengths and the ring dimensions to the appropriate values.

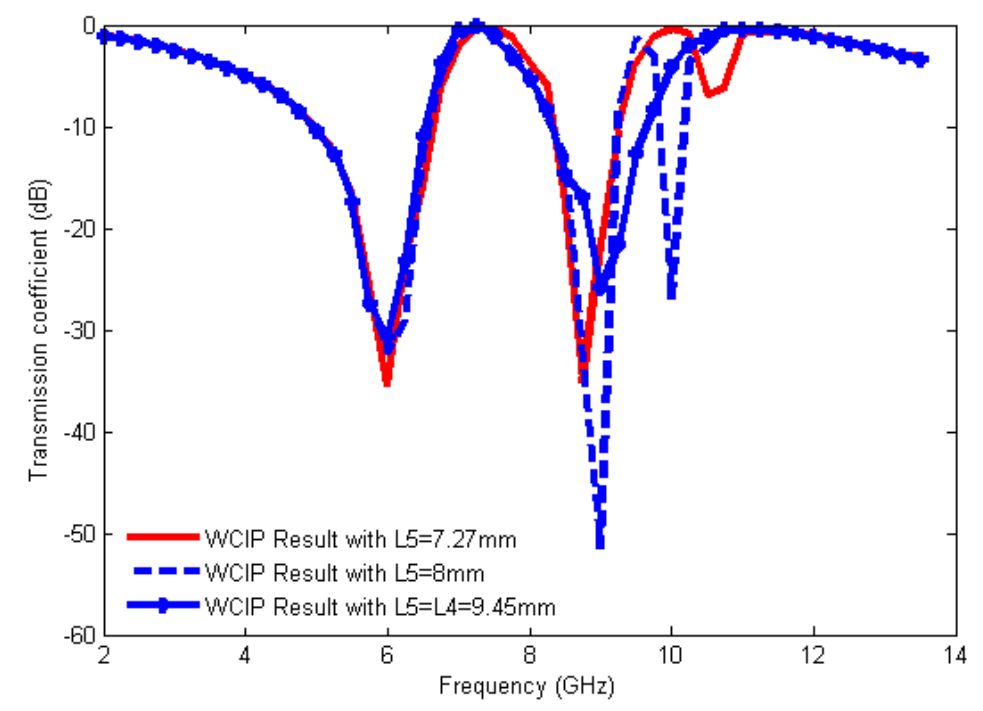

Fig. 16. Transmission coefficient for different increasing values of L5 for $y$-Polarization

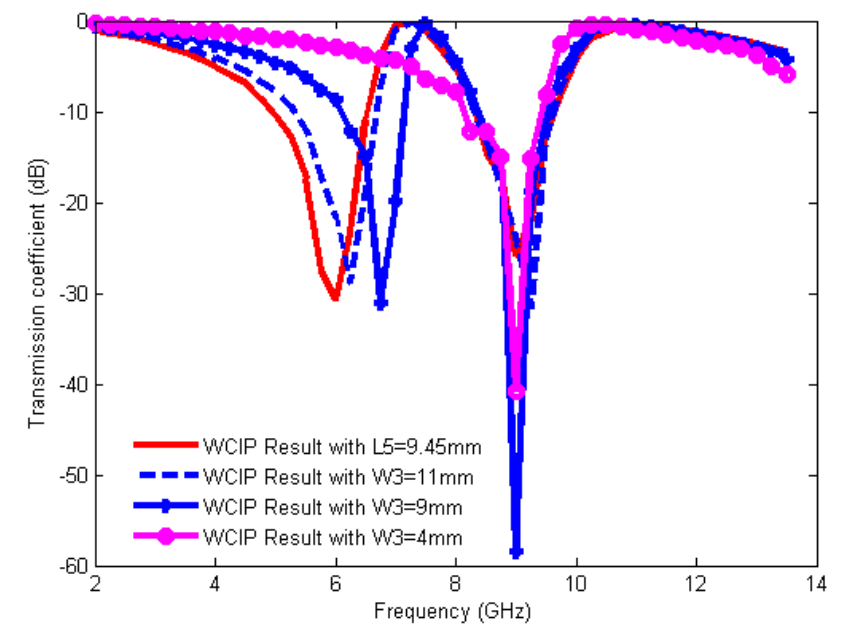

Fig. 17. Transmission coefficient for different decreases values of $W_{3}$ and fixed $L_{5}=9.45 \mathrm{~mm}$ for $y$-Polarization

Brazilian Microwave and Optoelectronics Society-SBMO Brazilian Society of Electromagnetism-SBMag received 08 Sep 2017; for review 08 Sep 2017; accepted 15 Mar 2018 (C) 2018 SBMO/SBMag
ISSN 2179 - 


\section{WCIP CONVERGENCE CRITERIA}

In the WCIP method the convergence is ensured independently of the metallic pattern at the interface [6]. The iterative procedure once the convergence is reached represented. The convergence is quantified by comparing the present iteration admittance to the previous iteration admittance once the admittance steady state is attained. A relative error of less than $10^{-2}$ is necessary to stop the iterative procedure and to provide precise results. In fact when the operating frequency is far away from the resonance the convergence is rapidly obtained and the number of necessary iterations decreases as getting far away from resonance. Thus the need of an adaptive algorithm to choose the number of needed iterations to reach convergence at each operating frequency is relevant leading to an important gain in computing time.

The input admittance $Y_{\text {in }}$ seen by the excitation source is calculated every iteration and at each operating frequency. The real and imaginary parts of the input admittance $Y_{\text {in }}$ are shown in figure 18 as a function of the iteration number for a given operating frequency where three different regions are present: transition region, chaotic region and convergence region.

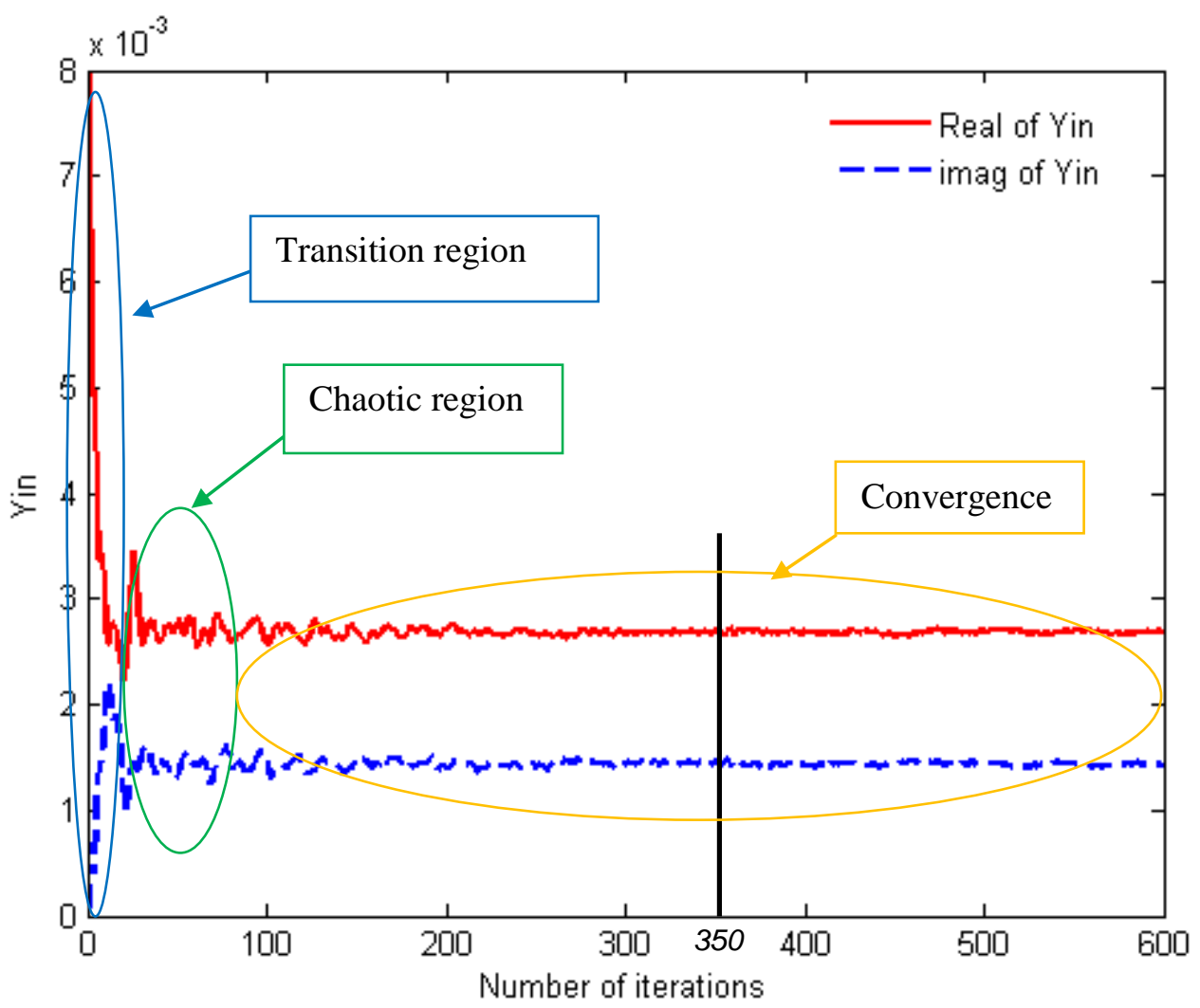

Fig. 18. Variation of Yin versus the number of iterations

The iterative procedure is stopped after 350 iterations. The relative error in the real part of the input admittance in the convergence region as compared to the previous iteration is calculated for $350^{\text {th }}$ Brazilian Microwave and Optoelectronics Society-SBMO received 08 Sep 2017; for review 08 Sep 2017; accepted 15 Mar 2018 Brazilian Society of Electromagnetism-SBMag 
iteration, $351^{\text {st }}$ iteration, $352^{\text {nd }}$ iteration and $353^{\text {rd }}$ iteration. The relative errors vary from $5.97 \times 10^{-3}$ to $8.21 \times 10-3$. It is expected to see the relative error decreasing but it is not the case because of the quasi oscillatory profile of the input admittance at the convergence region. To overcome this drawback, the mobile average technique which is an average of the input admittance over a range of 50 iterations for example is to be adopted. This technique permits smoothing the curve by filtering the oscillations and as a result the relative error will be decreasing from one iteration to the next iteration in the convergence region.

\section{CONCLUSION}

In this work a rectangular metallic ring FSS with non coupled parallel metallic strips for three bands and dual polarization applications is simulated and manufactured. In the proposed FSS type the strips and the rectangular ring making the FSS unit cell are independent in the spatial domain and also independent in the spectral domain since the metallic elements are uncoupled for a determined arrangement. This characteristic offers to the users a comprehensive FSS behavior at any resonant frequency of their frequency response since each resonant frequency is only due to one metallic FSS element intervening by only one dimension. Moreover its resonance is no more than an equivalent resonant LC circuit.

One of the fabricated structures presents three resonant frequencies at $6 \mathrm{GHz}, 8.25 \mathrm{GHz}$, and 10.5 $\mathrm{GHz}$ with bandwidths of $1.53 \mathrm{GHz}, 1.25 \mathrm{GHz}$, and $0.21 \mathrm{GHz}$ respectively when the incident wave is x-polarized and three resonant frequencies at $6 \mathrm{GHz}, 8.75 \mathrm{GHz}$, and $10.5 \mathrm{GHz}$ with bandwidths of $1.71 \mathrm{GHz}, 0.93 \mathrm{GHz}$ respectively when the source is y polarized. The resonant frequencies can be independently adjusted by the variation of the metallic strips lengths. For switchable FSS the insertion of a PIN diode switch at the center of the strips allows the insertion or the removal of the corresponding resonant frequency. Three FSS structures are fabricated and characterized. The WCIP results are in a good agreement with COMSOL Multiphysics and measurements.

The presented FSS is characterized by bandwidths that can be improved until more than $122 \%$ in both $x$ and $y$ polarized source directions by independently varying the strips lengths and the ring dimensions. Moreover, the same way can lead to tuning the resonant frequency centering the enhanced bandwidth.

\section{REFERENCES}

[1] R. Bhattacharyya, et al., "A Compact Frequency Selective Surface with Multi Frequency Operation", International Journal of Electronics and Communication Engineering, Vol. 9, No. 1, pp. 39-44, 2016.

[2] A. G. Neto, et al., “ Analysis of Frequency Selective Surface with U-Shaped Geometry”, JMO Journal, Vol. 14, pp. 113-122, July 2015,.

[3] Guo, H. Sun, et al., “A Novel Dual band Frequency Selective Surface With Periodic Cell Perturbation”, Progress In Electromagnetics Research B, Vol. 9, pp. 137-149, 2008. 
[4] Y. Li, et al., "Design And Synthesis of Multilayer Frequency Selective Surface Based on Antenna-Filter-Antenna Using Minkowski Fractal Structures", IEEE Transactions On Antennas And Propagation, Vol. 63, No. 4, pp. 133141, January 2015.

[5] H. Baudrand, M. Titaouine and N. Raveu, The Wave Concept in Electromagnetism and Circuits: Theory and Applications, ISTE Ltd and John Wiley \& Sons Inc., August 2016.

[6] A. Djouimaa et al., "Tunable FSS simulation using WCIP method for multiband and dual polarized application", Radioelectronics and Communications Systems, Vol. 60, No. 3, pp. 106-112, 2017.

[7] I. Adoui, et al., "Characterization Of Novel Open Notched Quasi-Square Metallic Ring FSS Using WCIP Method For Multiband Applications", Microwave And Optical Technology Letters. Vol. 58, No. 9 pp. 2071-2075, 2016, doi: $10.1002 / \mathrm{mop}$.

[8] M. AOUISSI, et al, "Analysis of a joined split-ring FSS structure characterized by three resonant frequencies and a tuned enhanced band using the WCIP method", Turkish Journal of Electrical Engineering \& Computer Sciences, Vol. 25,pp. 288- 2896, 2017.

[9] I. Adoui, M. Titaouine, A.Djouimaa, H. Choutri and H. Baudrand, "WCIP Method Applied to Modeling a Modified Rectangular Metallic Ring FSS for Multiband Applications", 7th seminar on Detection Systems: Architectures and Technologies (DAT'2017), 2017.

[10] M. Titaouine, et al., "Dual- Band And Enhanced Band FSS Characterization Using Wcip Method", MOTL Journal, Vol. 52, No. 4, pp. 836-839, April 2010.

[11] A. Djouimaa, M.Titaouine, H. baudrand, "Analysis of Double layered Frequency selective surface based on WCIPSM Technique", 7th seminar on Detection Systems: Architectures and Technologies (DAT'2017), 2017.

[12] M. Titaouine, et al., "Analysis of Frequency Selective Surface on Isotropic/Anisotropic Layers Using WCIP Method”, ETRI Journal, Vol. 29, No. 1, pp. 36-44, February 2007.

[13] M. Titaouine, et al., "Determination Of Metallic Ring Fss Scattering Characteristics Using WCIP Method", MOTL Journal, Vol. 50, No. 5, pp. 1324-1328, May 2008.

[14] M. Titaouine, et al., "Analysis of Shorted Ring Slots Frequency Selective Surfaces Using WCIP Method", JMOEA Journal, Vol. 07, No. 2, pp. 65-82, December 2008.

[15] V. P. Silva Neto, et al., "Wave Concept Iterative Procedure Analysis of Patch Antennas on Nanostructured Ceramic Substrates", ADVANCED Electromagnetics Journal, Vol. 02, No.3, pp. 41-44, December 2013.

[16] M. Ayari, et al., "An Extended Version of Transverse Wave Approach (TWA) for Full-Wave Investigation of Planar Structures”, JMO Journal, Vol. 07, No.2, pp. 123-138, December 2008.

[17] M. Tellache, et al., "An Numerical Method Based Iterative Process To Characterize Microwave Planar Circuits", Computing Journal, Vol. 07, pp. 86-94, 2008.

[18] S. Aroussi, et al., "Efficient Analysis of Complex FSS Structure Using the WCIP Method", Journal Of Electromagnetic Analysis And Applications, Vol. 3, pp. 447-451, 2011.

[19] G. Ramzi, et al., "Tunable Lowpass Filters Using Folded Slots Etched In The Ground Plane”, Progress In Electromagnetics Research C, Vol. 7, pp. 65-78, 2009.

[20] M. Titaouine, et al., "WCIP Method Applied to Active Frequency Selective Surface", Journal of Microwaves and Optoelectronics, Vol. 6, No. 1, pp. 1-16, June 2007.

[21] A. Sassi, et al., "Analysis and design of planar FSS circuits of approach multiscale combined with an auxiliary source technique using an Iterative Method", International Journal of Applied Engineering Research , Vol. 11, No. 12, pp. 7851-7856, 2016.

[22] A. E. Martynyuk, J. I. Martinez Lopez and N. A. Martynyuk, "Active frequency-selective surfaces based on loaded ring slot resonators", Electronics Letters, vol. 41, No. 1, pp. 2-4, January 2005. 